\title{
LEED ANALYSIS OF A DENSE LEAD MONOLAYER ON COPPER (100)
}

\author{
W. HÖSLER * and W. MORITZ \\ Institut für Kristallographie und Mineralogie der Universität München, Teresienstrasse 41, \\ D-8000 München 2, Fed. Rep. of Germany
}

Received 30 December 1985; accepted for publication 4 April 1986

\begin{abstract}
A LEED intensity analysis is reported for the $c(5 \sqrt{2} \times \sqrt{2}) \mathrm{R} 45^{\circ}$ structure, which is formed by a dense lead monolayer on the (100) surface of copper. Evidence was found that the adsorbate atoms do not arrange pseudo-hexagonally (i.e. without sixfold intra-layer coordination) as expected for a dense two-dimensional package. It was confirmed that the lead atoms still tend to occupy the hollow sites of the (100) surface and arrange in the narrow domains of a strained $c(2 \times 2)$ structure. These domains are regularly intersected by dislocation lines, so that adjacent domains are in antiphase position. Within this arrangement the adsorbate atoms are mutually equidistant with closer spacings than in bulk lead. The growth of the monolayer and the epitaxial growth of lead on copper(100) in Stranski-Krastanov mode are correlated to this structure.
\end{abstract}

\section{Introduction}

Metallic monolayers, which are condensed on single crystal faces of other metals, are of fundamental interest in surface science, since they represent quasi-ideal systems for atomic and electronic studies of static and dynamic behaviour in two dimensions. These systems find technical application in surfaces with specifically modeled properties, as e.g. chemical passivity, catalytic activity or enhanced electron emission. Metallic monolayers also represent the initial stage of crystallization, which affects the behaviour of further condensed adsorbate, i.e. the growth and stability of epitaxial multilayers.

In addition to simple structures at lower coverages, the complete monolayers of many heterogeneous metallic systems exhibit large unit cells (cf. e.g. ref. [1]). The complexity of these monolayer structures opposes any calculation of LEED intensities - a prerequisite for a detailed structure analysis - so that the atomic arrangement within the large cells is still a subject of discussion. The many plausible models, which have been suggested for these systems, still lack unambiguous confirmation.

The structural models which are applied to these surfaces, can be roughly separated into two groups, depending on the assumed predominant interac-

* Present address: Siemens AG, ZT ZFE FKE 41, Otto Hahn Ring 6, D-8000 München 83, Fed. Rep. of Germany. 
tions. The predominant interactions determine not only the static structure but also the thermodynamic behaviour of the adsorbed layer. For example twodimensional order-disorder transitions can be assumed to occur either by a lattice-gas-like behaviour or by a free two-dimensional mobility, two alternative approaches which are statically represented by the following geometric models.

On the assumption of dominant adsorbate-substrate bonds, the sites of the adsorbate atoms and hence their spacing are essentially locked to the periodicity of the substrate. The misfit strain, which is imposed on the adsorbed layer, can be released by a regular arrangement of dislocations, thus forming the observed superstructures. This dislocation model is represented by the calculations of Frank, van der Merwe and others [2-4] who confirmed, on the assumption of idealized interactions, that corresponding configurations are of minimal energy for one- and two-dimensional substrate-overlayer systems.

If, on the other hand, the interactions within the absorbed layer far exceed any localizing bonds to the substrate, the atoms of this layer will arrange in mutual sixfold coordination at an equilibrium spacing, which essentially disregards the substrate periodicity. Consequently the superstructure is defined by the coincidence of both lattices. This model is commonly applied to weakly bond adsorbates like noble gases on graphite or metals on metallic substrates. It has also been suggested for the reconstruction of the (100) faces of the noble metals and was confirmed for $\operatorname{Ir}(100)[5,6]$, which proved to form a topmost layer similar to a strained fcc (111) plane, lying on non-reconstructed fourfold (100) planes of the bulk.

The purpose of this study was to investigate whether the results for the noble metal also apply to a simple heterogeneous metallic adsorption system, or whether the arrangement of the adsorbate reflects the lateral variation of the adsorption potential, as predicted by the dislocation model. The structure of lead monolayers on Cu(110) has been investigated by LEED [7] and X-rays [8] with the result that in the strongly corrugated [100] direction the periodicity of the substrate dominates while along the troughs in [110] direction the substrate potential seems to be rather smooth and lateral interactions between lead atoms prescribe the periodicity of the superstructure. Nevertheless, the influence of the substrate potential in [110] direction is strong enough, giving rise to an incommensurate melting transition. In view of these results it is interesting to know which atomic arrangement exists in dense monolayers of lead on the smooth $(100)$ surface. At a coverage of 0.6 a well ordered $(5 \sqrt{2}$ $\times \sqrt{2}) \mathrm{R} 45^{\circ}$ superstructure is formed for which two different structural models corresponding to both alternative approaches described above have been proposed $[7,9]$. We present here the results of a structure refinement confirming previous results [10] which gave strong evidence for the dislocation model.

Another feature focused our attention on the dense monolayer on copper (100). Lead grows on this face in Stranski-Krastanov mode [7], i.e. 
additionally adsorbed lead forms three-dimensional nuclei, which remain surrounded by the monolayer until they coalesce at much higher coverage. This growth mode is microscopically the least understood, but the structure of the dense monolayer has to be regarded to play an essential role in the inhibition of the growth of the covering layers.

In the following two sections we briefly describe the experimental set-up and give some details of the LEED calculations. In section 4 we sketch the behaviour of lead on copper (100) at submonolayer coverages and outline the possible models of the monolayer structure. The results of the LEED analysis are presented in section 5 and the confirmed model is discussed there. The monolayer growth and the further growth in Stranski--Krastanov mode is related to these results in section 6 .

\section{Experiments}

The experiments were performed in a standard UHV chamber at pressures below $10^{-10}$ Torr. The chamber was equipped with LEED optics, including a movable Faraday cup, auxiliary electron gun for Auger spectroscopy and the common facilities for alignment, ion bombardment, heating and cooling of the sample. The copper single crystal was cleaned by cycles of argon ion sputtering and successive annealing, until the LEED beams had become sharp and brilliant. All impurities were below the detection limit of the Auger system 0.02 and 0.01 physical monolayers of sulphur and carbon, the main contaminants. The state and the alignment of the surface were checked by comparing the intensity spectra of the clean surface to calculated intensities. The agreement was good, depicted by an averaged Zanazzi-Jona $r$-factor below 0.04, which is comparable to the best results obtained for this surface [11].

The lead was evaporated from an RF-heated crucible. During evaporation the pressure increase was below $10^{-11}$ Torr and no contamination could be detected in the adsorbed layer. The superstructure was annealed by heating the sample to about $600 \mathrm{~K}$, the melting point of the monolayer, resulting, after cooling to ambient temperature, in an essential increase of the contrast in the LEED pattern. The incoherent background was further decreased by cooling the sample to $160 \mathrm{~K}$.

Under control of a mini-computer, the LEED beam currents were measured by a step-motor-driven Faraday cup. In order to obtain relative intensities, these currents were normalized to the primary beam current. Then the background was subtracted, having been determined during a separate run in the vicinity of the respective beam. The spectra of at least two symmetrically equivalent beams were averaged, in order to increase the reliability of the data. All spectra were taken several times after separate surface preparations to ensure reproducibility. 


\section{Calculations}

The LEED calculations were performed using the layer doubling scheme for inter-layer wave propagation [12] and the matrix inversion method for intra-layer scattering [13]. The calculations were done using the oblique primitive unit cell of the overlayer which contains three lead atoms and applying the full $\mathrm{cmm}$ symmetry in angular momentum space as described elsewhere [14]. The number of phase shifts was increased to 10, which has been proved previously to be sufficient for lead up to energies of about $230 \mathrm{eV}$. The potentials for copper and lead were obtained from a relativistically calculated free atom charge density renormalised by overlapping the contributions from atoms arranged in the fcc and $\mathrm{c}(2 \times 2)$ configuration, respectively. A local density exchange correction was applied. A detailed analysis of the influence of the potential model performed for the $\mathrm{c}(2 \times 2)$ structure showed very little influence on the structural parameters [15] and we expect the same to be the case for the $(5 \sqrt{2} \times \sqrt{2}) \mathrm{R} 45^{\circ}$ superstructure.

For clean copper an energy-dependent real part of the inner potential was derived [15] and proved to be in good accordance with comparable measurements $[16,17]$ and theory [18]. The energy dependence of the real part of the inner potential used in the intensity analysis was $V_{0 r}(E)=-3.9-57.8(E+$ $12.0)^{-1 / 2} \mathrm{eV}$, where the correction due to the work function of the tungsten cathode is not included. This inner potential could also be employed for the adsorbate system with no need of corrections. The $r$-factors defined by Zanazzi and Jona [19] and that suggested by Pendry [20] were used for data evaluation, and the visual comparison of the spectra served as a complementary tool for the evaluation of the models. 21 symmetrically inequivalent beams with a total energy range of $2150 \mathrm{eV}$ were the data base for the structure analysis.

During preliminary calculations the Debye temperature of the adsorbed lead was varied between 50 and $220 \mathrm{~K}$. The isotropic Debye temperature of the topmost atoms is of ambiguous physical meaning, but was used here as a rough non-structural fitting parameter to improve the reliability of the derived structure. The $r$-factors exhibited two different elongated minima around $85 \mathrm{~K}$ (Zanazzi-Jona) and $140 \mathrm{~K}$ (Pendry), due to emphasis on different details of the spectra. All succeeding calculations were performed with an intermediate value of $120 \mathrm{~K}$ for lead, while for the copper substrate the bulk value of $330 \mathrm{~K}$ was used.

Three geometric parameters of the lead layer were varied for each model, one lateral shift and two vertical spacings as described in the following. An influence on the substrate was additionally considered by variation of the topmost copper layer spacing. 


\section{Models}

When lead is condensed on the clean (100) surface of copper, after an initial increase of background, beams of a $c(4 \times 4)$ superstructure become visible in the LEED pattern. During increasing coverage these beams gain a maximum of intensity, followed by a diminution, until a well expressed $\mathrm{c}(2 \times 2)$ structure is depicted by intense half-order beams. The LEED analysis confirmed that this structure is formed by lead atoms in the fourfold hollow sites at a lead copper spacing which is about $4 \%$ closer than the sum of both metallic radii $[10,15]$. With further increasing coverage the half-order beams become rapidly diffuse and split into satellites which later on move to the beam positions of the $\mathrm{c}(5 \sqrt{2} \times \sqrt{2}) \mathrm{R} 45^{\circ}$ superstructure.

The lead coverage of the monolayer structure was determined by evaluating the heights of the 90 and $94 \mathrm{eV}$ lead Auger peaks and by normalizing them to those of the $c(2 \times 2)$ structure. The latter structure is very sensitive to slight deviations of the coverage, thus yielding a good standard for calibration. By attributing a crystallographic coverage of 0.5 to this superstructure, the coverage of the $c(5 \sqrt{2} \times \sqrt{2}) \mathrm{R} 45^{\circ}$ structure was determined to 0.6 . (Contrary to the supposition of a second $\mathrm{c}(5 \sqrt{2} \times \sqrt{2}) \mathrm{R} 45^{\circ}$ structure at lower densities [21], ordered $c(5 \sqrt{2} \times \sqrt{2}) \mathrm{R} 45^{\circ}$ structures were never observed in any other range of coverage.)

The observed LEED pattern of the $\mathrm{c}(5 \sqrt{2} \times \sqrt{2}) \mathrm{R} 45^{\circ}$ structure (fig. 1) originates from two domains, each one of cmm symmetry and inclined $90^{\circ}$ relative to the other. The lead coverage of 0.6 corresponds to six lead atoms, occupying the centered unit cell. Taking into account that the width of the cell ( $3.615 \AA$ ) is virtually equal to the diameter of bulk metallic lead ( $3.50 \AA)$ and following standard tables [22], the $\mathrm{cmm}$ symmetry tolerates only two distinct atomic intra-layer arrangements (fig. 1). These two arrangements correspond to the two alternative approaches mentioned above; either dominant adsorbate-adsorbate or dominant adsorbate-substrate interactions.

One arrangement - often proposed for dense overlayers - shows a mutual sixfold intra-layer coordination and usually is termed "hexagonal". Since any hexagonal symmetry obviously is upset by the fourfold substrate, this arrangement will be called "pseudo-hexagonal" hereafter. The other arrangement is characterized by a row of atoms in fourfold coordination consecutively followed by two rows in fivefold coordination. This model is equivalent to a system of narrow domains of $\mathrm{c}(2 \times 2)$ structure, regularly intersected by parallel dislocation lines, so that adjacent regions are in antiphase position. This arrangement will be termed the "antiphase"-model.

There are two possibilities placing the $\mathrm{cmm}$ layer onto the $4 \mathrm{~mm}$ substrate and retaining a total $\mathrm{cmm}$ symmetry. The origin of the $\mathrm{cmm}$ unit cell has to be placed either in a hollow site between four copper atoms or on top of a copper atom, resulting in a duplication of the possible models (cf. fig. 1). For each 

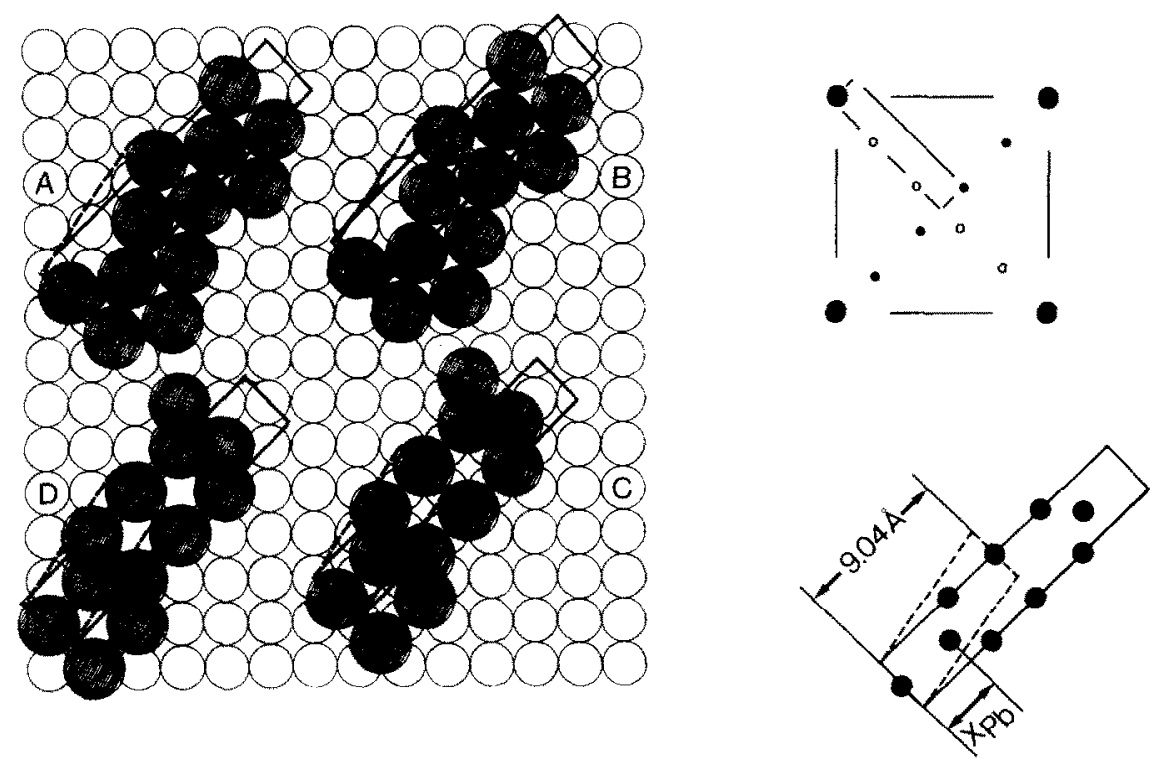

Fig. 1. The four models of the $\mathrm{Cu}(100)-\mathrm{c}(5 \sqrt{2} \times \sqrt{2}) \mathrm{R} 45^{\circ}-\mathrm{Pb}$ structure: pseudo-hexagonal hollow site model (A), pseudo-hexagonal top site model (B), $\mathrm{c}(2 \times 2)$ hollow site antiphase domains (C) and the same domains in top site (D), where lead atoms are shown with bulk metallic radius. These four models correspond to the two possible cmm intra-layer arrangements in two positions, relative to the substrate. Also shown is a schematic cell of the LEED pattern, where the integral-order beams are emphasized in bold and the beams, originating from the rotated domain, are indicated by open circles. The centered and the primitive unit cell together with the parameter $X_{\mathrm{pb}}$ for the lateral spacing between lead atoms as used in figs. 3-5 is additionally given.

model the symmetry tolerates the variation of three geometric parameters of the adsorbate layer - one shift parameter which affects the lateral position of the four lead atoms with local non-4 mm-symmetry (fig. 1) and the spacings of the two geometrically inequivalent positions to the topmost copper layer.

The pseudo-hexagonal hollow site model was suggested by Henrion and Rhead after their observation of the $\mathrm{c}(5 \sqrt{2} \times \sqrt{2}) \mathrm{R} 45^{\circ}$ structure [7]. Biberian and Huber preferred the antiphase hollow sitc model [9], following Huber and Oudar, who had suggested corresponding "high symmetry models" for a number of comparable systems [23]. The top site antiphase model, which is composed of domains of top site $c(2 \times 2)$ structure, can be ruled out here, since the hollow sites were confirmed unequivocally for the $c(2 \times 2)$ structure $[10,15]$. Thus the pseudo-hexagonal top site model gains more probability, since it places two of three lead atoms close to the hollow sites, so that in total three of the four models from above had to be considered in the calculations. 


\section{Results and discussion}

The spectra which were calculated for the pseudo-hexagonal hollow site model of the common form, i.e. with equidistant adsorbate atoms, do not show any agreement with the measured spectra. The very poor $r$-factors (cf. table 1) and the lack of visual similarities between the spectra (curve A in fig.

Table 1

Summary of the results

\begin{tabular}{|c|c|c|c|c|c|c|c|}
\hline \multirow[t]{2}{*}{ Model } & \multicolumn{5}{|l|}{ Parameters } & \multicolumn{2}{|c|}{$r$-Factors } \\
\hline & $x$ & $\begin{array}{l}d_{\mathrm{Pb}} \\
(\AA)\end{array}$ & $\begin{array}{l}\Delta d_{\mathrm{Pb}} \\
(\AA)\end{array}$ & $\begin{array}{l}\Theta_{\mathrm{Pb}} \\
(\mathrm{K})\end{array}$ & $\begin{array}{l}d_{\mathrm{Cu}} \\
(\AA)\end{array}$ & $r_{\mathrm{P}_{\min }}$ & $r_{\mathrm{ZJ}_{\min }}$ \\
\hline \multirow{5}{*}{$\begin{array}{l}\text { Hexagonal } \\
\text { hollow site }\end{array}$} & 0.333 & 2.10 to 2.50 & -0.2 to 0.6 & 120 & 1.81 & \multirow{3}{*}{0.89} & \\
\hline & 0.333 & 2.42 & 0.34 & 120 & 1.81 & & \\
\hline & 0.333 & 2.31 & 0.42 & 120 & 1.81 & & 0.525 \\
\hline & 0.333 & 2.19 & 0.45 & 120 & 1.81 & \multirow[t]{2}{*}{0.89} & \\
\hline & 0.333 & 2.29 & -0.04 & 120 & 1.81 & & 0.525 \\
\hline \multirow{3}{*}{$\begin{array}{l}\text { Hexagonal } \\
\text { hollow site } \\
\text { (fig. 3) }\end{array}$} & 0.20 to 0.36 & 2.10 to 2.50 & 0.0 & 120 & 1.61 to 2.01 & \multirow{3}{*}{0.70} & \\
\hline & 0.261 & 2.30 & 0.0 & 120 & 1.80 & & \\
\hline & 0.251 & 2.29 & 0.0 & 120 & 1.81 & & 0.385 \\
\hline \multirow{3}{*}{$\begin{array}{l}\text { Hexagonal } \\
\text { hollow site }\end{array}$} & 0.26 & 2.10 to 2.50 & -0.2 to 0.6 & 120 & 1.81 & \multirow{3}{*}{0.70} & \\
\hline & 0.26 & 2.25 & -0.2 to 0.6 & 120 & 0.06 & & \\
\hline & 0.26 & 2.29 & 0.0 & 120 & 0.06 & & 0.385 \\
\hline \multirow{3}{*}{$\begin{array}{l}\text { Hexagonal } \\
\text { top site } \\
\text { (fig. 4) }\end{array}$} & 0.325 to 0.370 & 2.10 to 2.50 & 0.0 & 120 & 1.61 to 2.01 & \multirow{3}{*}{0.68} & \\
\hline & 0.357 & 2.29 & 0.0 & 120 & 1.85 & & \\
\hline & 0.355 & 2.32 & 0.0 & 120 & 1.83 & & 0.350 \\
\hline \multirow{3}{*}{$\begin{array}{l}\text { Hexagonal } \\
\text { top site }\end{array}$} & 0.355 & 2.10 to 2.50 & -0.2 to 0.6 & 120 & 1.81 & \multirow{3}{*}{0.68} & \\
\hline & 0.355 & 2.30 & 0.01 & 120 & 1.81 & & \\
\hline & 0.355 & 2.34 & -0.01 & 120 & 1.81 & & 0.360 \\
\hline \multirow{3}{*}{$\begin{array}{l}\text { Antiphases } \\
\text { (fig. 5) }\end{array}$} & 0.355 & 2.10 to 2.50 & 0.0 & 50 to 220 & 1.61 to 2.01 & \multirow{3}{*}{0.46} & \\
\hline & 0.355 & 2.32 & 0.0 & 140 & 1.81 & & \\
\hline & 0.355 & 2.31 & 0.0 & 85 & 1.83 & & 0.225 \\
\hline \multirow{3}{*}{$\begin{array}{l}\text { Antiphases } \\
\text { (fig. 5) }\end{array}$} & 0.340 to 0.385 & 2.10 to 2.50 & 0.0 & 120 & 1.61 to 2.01 & \multirow{3}{*}{0.46} & \\
\hline & 0.354 & 2.32 & 0.0 & 120 & 1.81 & & \\
\hline & 0.360 & 2.32 & 0.0 & 120 & 1.83 & & 0.235 \\
\hline \multirow{3}{*}{$\begin{array}{l}\text { Antiphases } \\
\text { (fig. 5) }\end{array}$} & 0.355 & 2.10 to 2.50 & -0.2 to 0.6 & 120 & 1.81 & \multirow{3}{*}{0.46} & \\
\hline & 0.355 & 2.31 & 0.02 & 120 & 1.81 & & \\
\hline & 0.355 & 2.32 & 0.0 & 120 & 1.81 & & 0.240 \\
\hline
\end{tabular}

The range of the varied parameters is given for each of the models in the first line, whereas succeeding lines show the depths of the $r$-factor minima and the corresponding set of parameters. The parameter $x$ defines the lateral position of two atoms in the oblique cell and is given in units of that supercell $(9.04 \AA), \Theta_{\mathrm{Pb}}$ represents the lead Debye temperature, $\Delta d_{\mathrm{Pb}}$ is the corrugation of the lead layer and $\mathrm{d}_{\mathrm{Cu}}$ is the top substrate layer spacing. The table omits various preceding calculations which yielded preliminary limits for these parameters. 

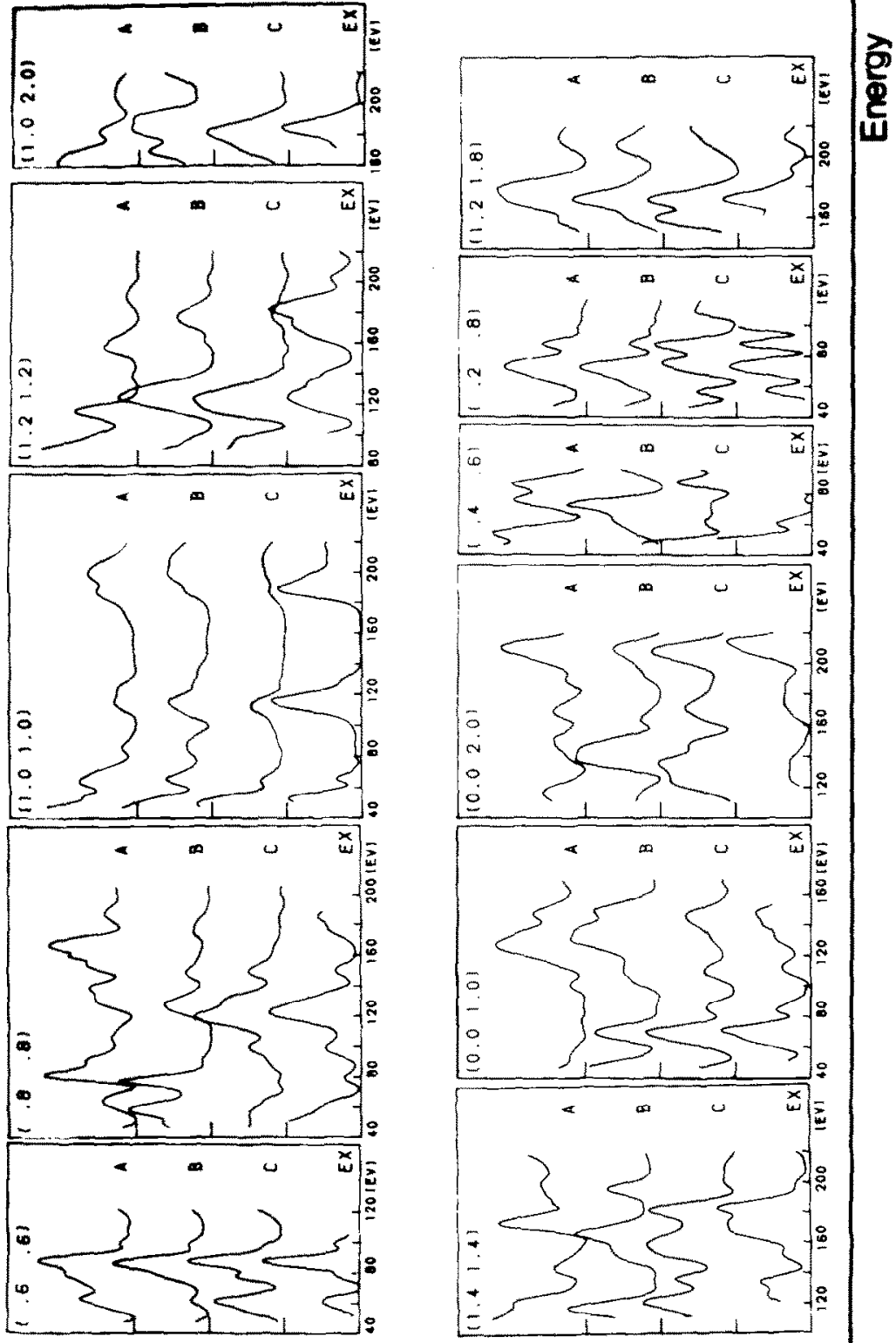

(sł!uก qu 

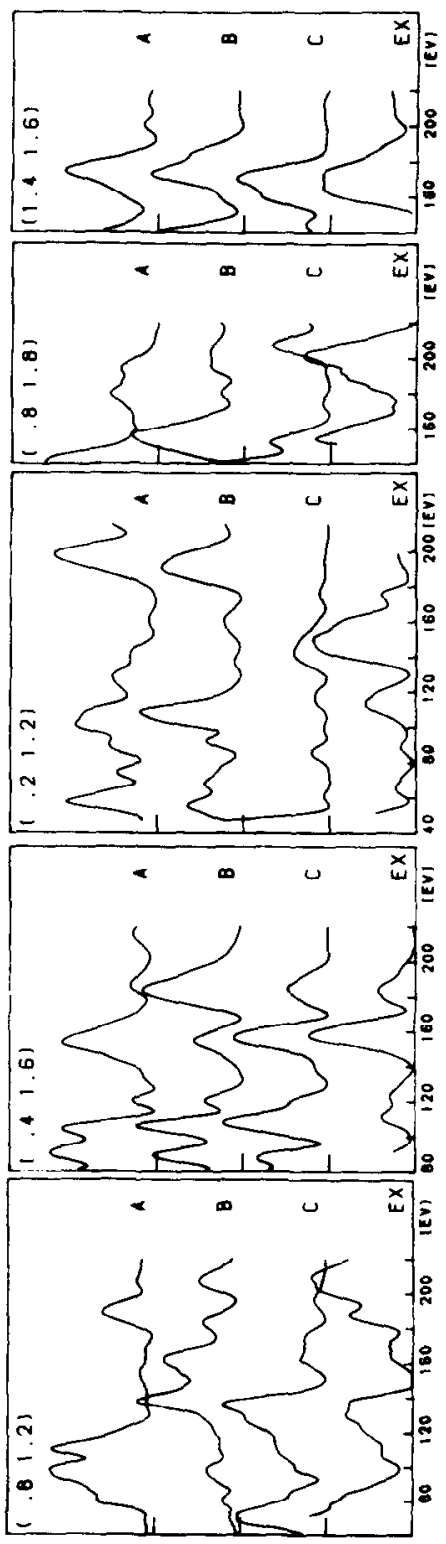

훙

2

ष

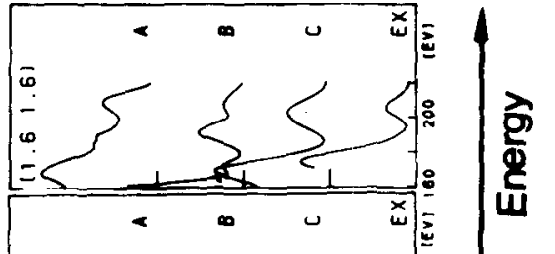

$\stackrel{0}{8}$

$8 \dot{x}$

西些

总

总 ${ }^{\circ}$

㱐

\& 0

के

है

穿

$\approx$ 政

苛哭

है

寻

$g$.

ㅎㅇㅇㅇㅇ

힘

흉

(a)

क्ष

言合

흥오음

픙

요용

胥

कै

字

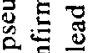

웡

음

명

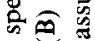

른

要

可

อ 氧

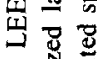

i

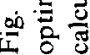




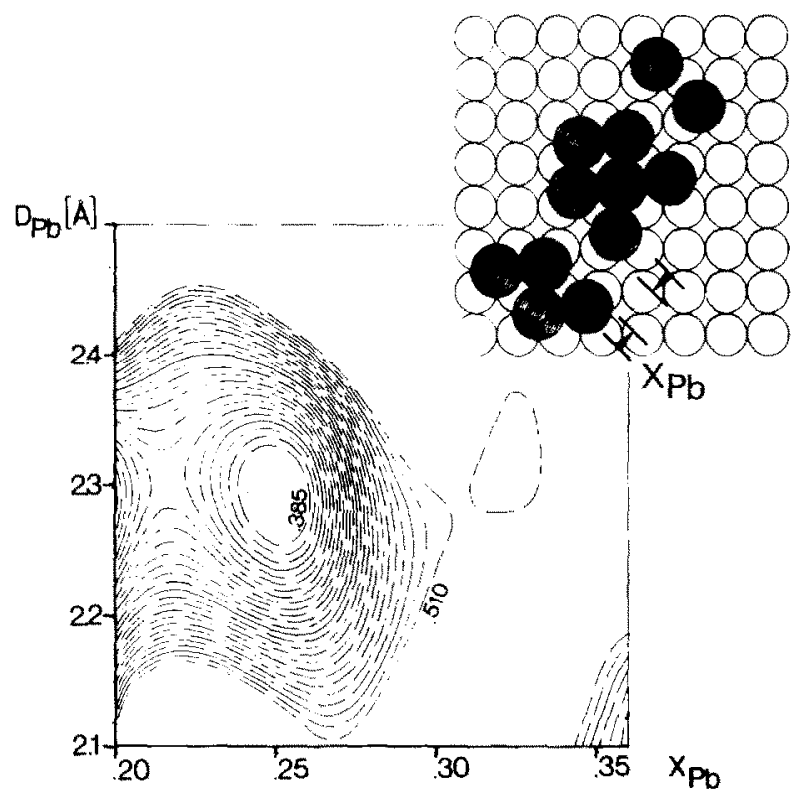

Fig. 3. Dependency of the Zanazzi-Jona $r$-factor on lateral shift and layer spacing $D_{\mathrm{Pb}}$ for the pseudo-hexagonal hollow site model. The inset shows the arrangement, which corresponds to the minimum of the $r$-factor. The lateral position $X_{\mathrm{Pb}}$ is given in units of the oblique supercell $(9.04$

$\AA)$, and the variation which is indicated in the model corresponds to that of the $r$-factor plot.

2) do not confirm a model of densely packed equidistant atoms, even with modified non-structural or structural parameters, such as Debye temperature, inner potential or corrugation. By rejecting equidistance and by varying the horizontal spacing between the lead atoms, the $r$-factors decreased to still poor values of 0.70 (Pendry) and 0.385 (Zanazzi-Iona). A lateral shift of the non-hollow site atoms, from the positions of equidistance, of $0.75 \AA$ towards the next hollow sites (cf. fig. 3) was necessary for this decrease, but again any accord with the spectra visually is hard to detect and a corrugation does not improve the $r$-factors. This lateral shift corresponds to next-neighbour spacings of $2.9,2.9$, and $4.9 \AA$ respectively - a surprising result for a structure which is assumed to be caused by nearest-ncighbour repulsion.

The minimal $r$-factors of the pseudo-hexagonal top site model are close to those of the hollow site model - 0.68 (Pendry) and 0.35 (Zanazzi-Jona) - but here the necessary lateral shift of the non-top site atoms towards the next hollow sites amounts to only $0.36 \AA$ and thus the resulting structure is closer to equidistancy (cf. fig. 4). For this model again, the agreement does not improve by a plausible buckling of the lead layer.

The pseudo-hexagonal top site model and the antiphase hollow site model differ mainly in the site of only one atom within the primitive oblique unit cell 


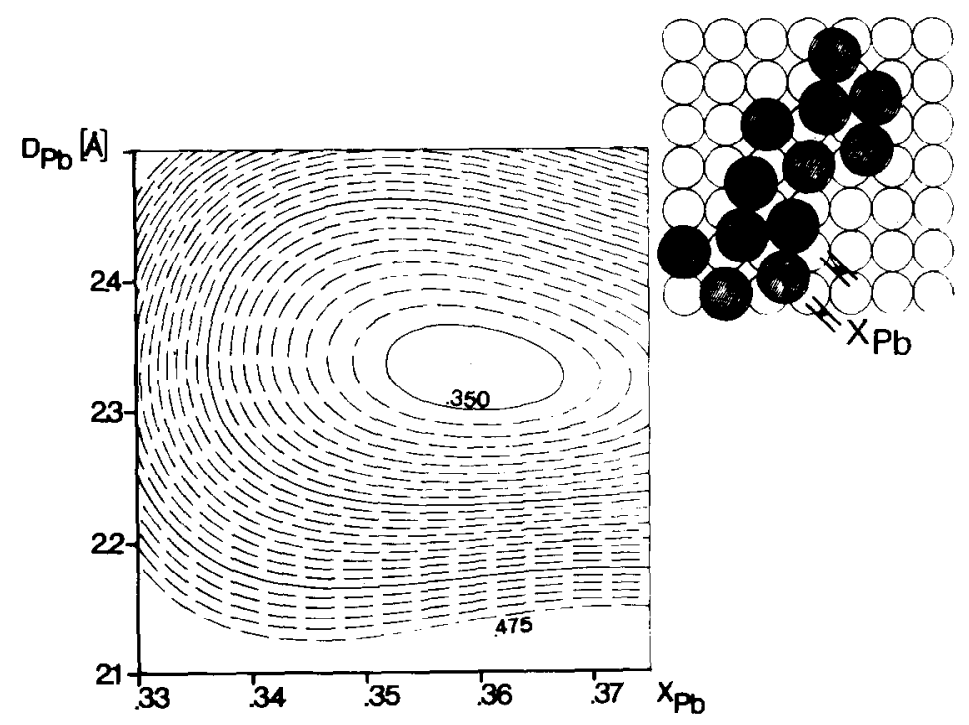

Fig. 4. Same as fig. 3 for the pseudo-hexagonal top site model.

apart from the slightly different lateral shifts (cf. figs. 1B and 1C). A mere shift of this atom to the respective site of the antiphase model, retaining all other parameters from the optimal hexagonal one, decreases the $r$-factors to values of 0.46 (Pendry) and 0.23 (Zanazzi-Jona) and increases significantly the accordance between the corresponding theoretical and the experimental spectra (fig. 2).

A detailed analysis based on the premise of an antiphase hollow site structure showed deep and narrow $r$-factor minima for each of the four geometric parameters (fig. 5). The resulting lead layer was very flat with a corrugation below $0.05 \AA$. Near the domain boundaries the lead atoms are shifted $0.40(10) \AA$ out of the hollow sites towards the domain mids. This value corresponds well to $0.42 \AA$, which would result for equidistant atoms in antiphase arrangement. The layer spacing amounts to $2.32(5) \AA$, which is equivalent to the $2.29(4) \AA$, obtained for the $c(2 \times 2)$ structure [15]. The spacing between the topmost two copper layers is $1.82(3) \AA$, slightly larger than the topmost layer spacing of the contracted clean (100) surface of copper $[10,11]$, but comparable to the (100) layer distance in bulk copper $(1.807 \AA)$ and to the topmost copper spacing which was found underneath the $\mathrm{c}(2 \times 2)$ lead structure [15].

The distances between lead and the nearest copper atoms are 2.94(4) and $2.72(10) \AA$ for hollow-site and near-hollow-site atoms, respectively, corresponding to lead radii of $1.66(4)$ and $1.44(10) \AA$. The latter radius bears some systematic uncertainty, for it is based on the spacing of one lead atom to only 


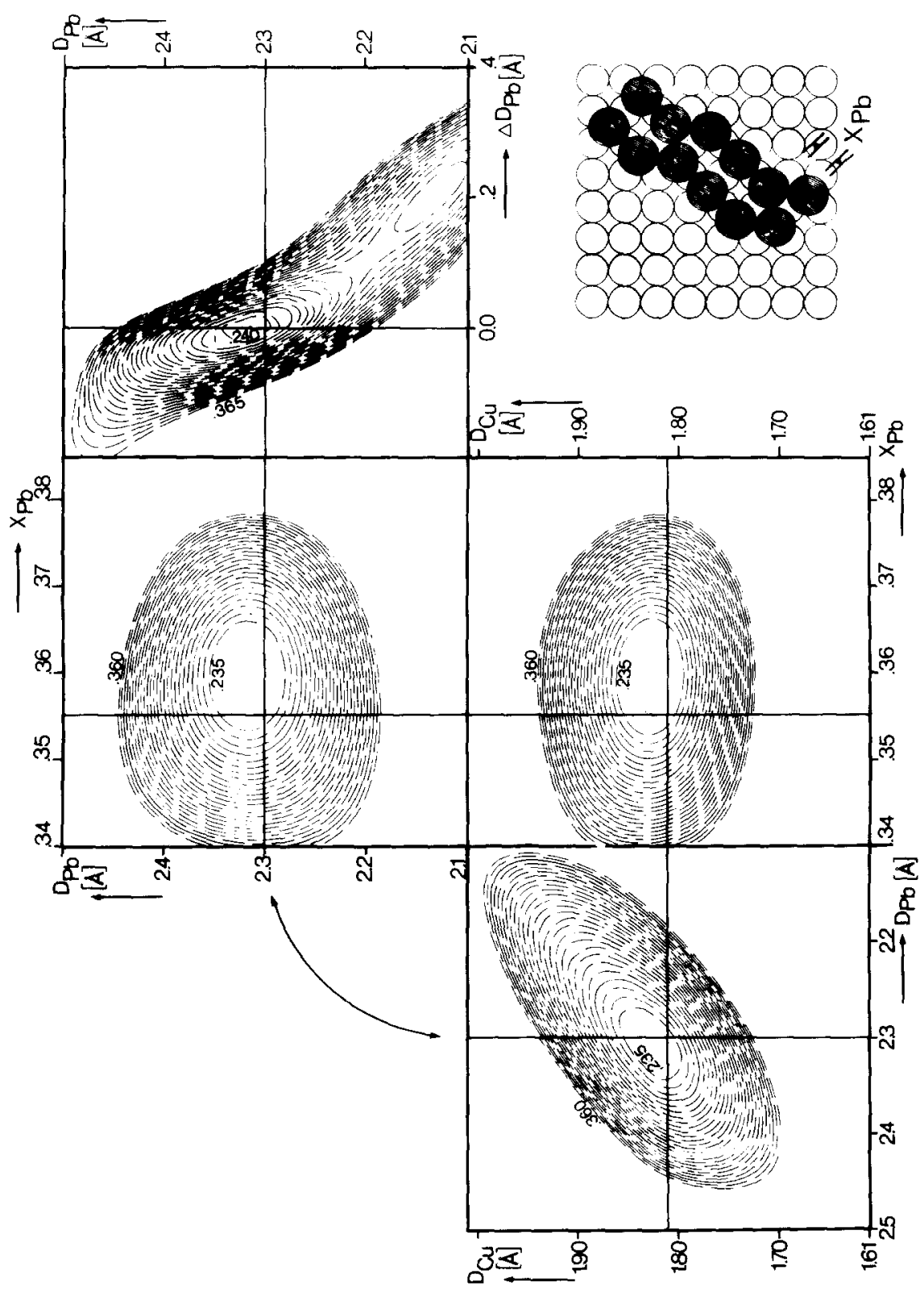

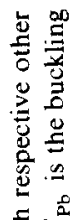

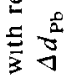
突 
one copper atom under the assumption of a non-reconstructed copper layer. This spacing is very sensitive to slight vertical or horizontal displacements of that one copper atom, so that a faint reconstruction, below the resolution limit of this LEED analysis, is able to eliminate the differences of the radii. A lead radius of $1.58(9) \AA$ results from the intra-layer spacing of the adsorbate, in good accordance with the first of the two values from above. Thus the radius of adsorbed lead is less than that of bulk metallic lead $(1.75 \AA)$, but comparable to reduced radii of lead, which were found in bulk binary alloys like $\mathrm{RhPb}_{2}, \mathrm{PdPb}_{2}$ and $\mathrm{AuPb}_{2}$ [24].

As pointed out above, the structural parameters and the sites of the confirmed antiphase hollow site model are reproduced by the hexagonal models of minimal $r$-factors to some extent. A lateral shift of about $0.4 \AA$ out of the hollow sites, the flat lead layer and a layer spacing of about $2.3 \AA$ is common to all three models. The antiphase model however yields $r$-factors, which are definitely lower than those of the other models. Thus the resulting antiphase structure is the actual cause of the weaker minima of the $r$-factors, which were observed for the pseudo-hexagonal models.

\section{Conclusions}

All results give evidence that the $c(5 \sqrt{2} \times \sqrt{2}) \mathrm{R} 45^{\circ}$ structure is not pseudo-hexagonal, i.e. there is no sixfold coordination within the adsorbate layer as would be assumed for densely packed atoms. Within the dense monolayer the adatoms still tend to occupy the fourfold sites of the substrate, in essence confirming laterally localized bonds, even for this simple metallic adsorption system. The dominant feature of the imposed monolayer structure is the $\mathrm{c}(2 \times 2)$ structure for one part, intersected by a regular arrangement of dislocations for the other part. From this, the transition from the $\mathrm{c}(2 \times 2)$ structure to the $\mathrm{c}(5 \sqrt{2} \times \sqrt{2}) \mathrm{R} 45^{\circ}$ structure is straightforward; during increasing coverage, dislocation lines are inserted into the $c(2 \times 2)$ structure and then rearrange two-dimensionally until their closest arrangement is established by the $c(5 \sqrt{2} \times \sqrt{2}) \mathrm{R} 45^{\circ}$ structure. This is visualized first by an increasing diffuseness of the half-order beams in the LEED pattern, followed by a splitting into satellites which later on move to the beam positions of the dense monolayer structure [10].

Another feature of interest is the impact of the $c(5 \sqrt{2} \times \sqrt{2}) \mathrm{R} 45^{\circ}$ structure on the further growth of lead on copper(100). Though the dense monolayer differs essentially from the (100) plane of lead, bulk lead crystallites are oriented on copper (100) with $\mathrm{Pb}(100) \| \mathrm{Cu}(100)$ and $\mathrm{Pb}[010] \| \mathrm{Cu}[011]$ [7]. The $\mathrm{c}(5 \sqrt{2} \times \sqrt{2}) \mathrm{R} 5^{\circ}$ structure can be retained at the bottom of $(100)$ oriented lead nuclei only, if the dislocations, originating from the interfacial structure and starting from there, do not propagate into the nuclei, but bend back and 
end again at the interface. Thus after a few layers the antiphase structure is upset so that regular $\mathrm{c}(2 \times 2)$ planes, which are very similar to $\mathrm{Pb}(100)$ planes, can develop. However this interfacial structure presumably is unstable, since the two-dimensional dislocations in the nuclei carry a large amount of strain energy (far exceeding that of the dislocation lines in the monolayer) and can easily dissolve by migration towards the interfacial plane. Consequently the $\mathrm{c}(5 \sqrt{2} \times \sqrt{2}) \mathrm{R} 45^{\circ}$ structure rearranges during further adsorption and the bottom plane of the nuclei is a $\mathrm{c}(2 \times 2)$ plane. Thus either the incorporated dislocations or - more probably the rearrangement of the basal plane raises the free enthalpy of lead multilayers - a necessary prerequisite for StranskiKrasanov growth, as it was pointed out by Bauer [25]. The total free enthalpy does not decrease before nuclei of stable size can be formed, which still remain surrounded by the monolayer.

Though the misfit dislocations, as calculated by Frank and van der Merwe [2], exhibit a dominant feature of the monolayer, these dislocations are not identical to those described by the van der Merwe model of epitaxial layer growth [26], since they are not necessary for a fit of the (100) planes of the different bulk metals. Contrary to that model, the dislocations of the $\mathrm{c}(5 \sqrt{2}$ $\times \sqrt{2}) \mathrm{R} 45^{\circ}$ structure oppose the growth of lead multilayers - in nuclei they have to be suppressed, which is the probable cause for the Stranski-Krastanov growth of lead on copper (100).

\section{Acknowledgements}

Financial support from the Deutsche Forschungsgemeinschaft via the Sonderforschungsbereich 128 is gratefully acknowledged. We are indebted to H. Jagodzinski for stimulating and helpful discussions. We express our thanks for the technical support, which was given by $\mathrm{H}$. Plöckl, J. Cziszar and R. Wunderlich.

\section{References}

[1] J.P. Biberian and G.A. Somorjai, J. Vacuum Sci. Technol. 16 (1979) 2073.

[2] F.C. Frank and J.H. van der Merwe, Proc. Roy. Soc. (London) A198 (1949) 205.

[3] J.H. van der Merwe, J. Appl. Phys. 41 (1970) 4725.

[4] J.A. Snyman and H.C. Snyman, Surface Sci. 105 (1981) 357.

[5] E. Lang, K. Müller, K. Heinz, M.A. Van Hove, R.J. Koestner and G.A. Somorjai, Surface Sci. 127 (1983) 347.

[6] W. Moritz, F. Müller and D. Wolf, to be published.

[7] J. Henrion and G.E. Rhead, Surface Sci. 29 (1972) 20.

[8] W.C. Marra, P.H. Fuoss and P.E. Eisenberger, Phys. Rev. Letters 49 (1982) 1169.

[9] J.P. Biberian and M. Huber, Surface Sci. 55 (1976) 259. 
[10] W. Hösler and W. Moritz, Surface Sci. 117 (1982) 196;

W. Hösler, Thesis, Universität München (1982).

[11] J.R. Noonan and H.L. Davis, in: Proc. ICSS-4 and ECOSS-3, Cannes, 1980, p. 1100;

II.L. Davis and J.R. Noonan, J. Vacuum Sci. Technol. 20 (1982) 842;

H.L. Davis and J.R. Noonan, Surface Sci. 126 (1983) 245.

[12] M.A. Van Hove and S.Y. Tong, Surface Crystallography by Low Energy Electron Diffraction (Springer, Berlin, 1979).

[13] J.B. Pendry, Low Energy Electron Diffraction (Academic Press, London, 1974).

[14] W. Moritz, J. Phys. C17 (1984) 353.

[15] W. Hösler, W. Moritz, E. Tamura and R. Feder, Surface Sci. 171 (1986) 55.

[16] J. Neve, P. Westrin and J. Rundgren, J. Phys. C16 (1983) 1291.

[17] S.A. Lindgren, L. Walldén, J. Rundgren and P. Westrin, Phys. Rev. Letters 50 (1983) 368;

S.A. Lindgren, L. Walldén, J. Rundgren and P. Westrin, Phys. Rev. B29 (1984) 576.

[18] L. Hedin and B.F. Lundquist, J. Phys. C4 (1971) 2064.

[19] E. Zanazzi and F. Jona, Surface Sci. 62 (1977) 61.

[20] J.B. Pendry, J. Phys. C13 (1980) 937.

[21] C. Argile, M.-G. Barthes-Labrousse and G.E. Rhead, Surface Sci. 138 (1984) 181.

[22] International Tables for X-Ray Crystallography (Kynoch, Birmingham, 1952).

[23] M. Huber and J. Oudar, Surface Sci. 47 (1975) 605.

[24] E.E. Havinga, H. Dansma and P. Hokkding, J. Less-Common Metals 27 (1972) 169.

[25] E. Bauer, Z. Krist. 110 (1958) 372.

[26] J.H. van der Merwe, Proc. Phys. Soc. (London) A63 (1950) 616. 